\title{
Magnetic Symmetry, Regge Trajectories and the Linear Confinement in Dual QCD
}

\author{
Hemwati Nandan • H.C. Chandola $\cdot$ H. Dehnen
}

Received: 16 September 2007 / Accepted: 29 October 2007 / Published online: 17 November 2007

(C) Springer Science+Business Media, LLC 2007

\section{Erratum to: Int. J. Theor. Phys. 44(4), 457-469 (2005)}

DOI 10.1007/s10773-005-3976-7

Some of the typographical mistakes in our abovementioned paper are corrected as follows:

1. Equation (6) should be read as:

$$
B_{\mu \nu}=-g^{-1} \sin \alpha\left(\partial_{\mu} \alpha \partial_{\nu} \beta-\partial_{\nu} \alpha \partial_{\mu} \beta\right) \equiv B_{\nu, \mu}-B_{\mu, \nu} .
$$

2. Equation (8) should be read as:

$$
£=-\frac{1}{4}\left|B_{\mu \nu}^{(d)}\right|^{2}+\left|\left(\partial_{\mu}+i 4 \pi g^{-1} B_{\mu}^{(d)}\right) \phi\right|^{2}-V\left(\phi^{*} \phi\right) .
$$

3. Equation (22) should be read as:

$$
H^{\prime \prime}+\frac{H^{\prime}}{r}-\frac{(n+K)^{2}}{r^{2}} H-H^{3} \ln H=0 .
$$

4. Equation (30) should be read as:

$$
J^{(m)}=2 \int_{0}^{\frac{R}{2}} d R_{0} \frac{E_{(m)}(K, H) v}{\left(1-v^{2}\right)^{\frac{1}{2}}}=I_{(m)} \frac{\alpha_{s}}{64} m_{B}^{2} R^{2} .
$$

The online version of the original article can be found under doi 10.1007/s10773-005-3976-7.

H. Nandan $(\bowtie)$

Indian Institute of Technology, Kharagpur, West Bengal 721 302, India

e-mail: hnandan@cts.iitkgp.ernet.in

H.C. Chandola

Department of Physics, Kumaun University, Nainital 263 001, India

H. Dehnen

Department of Physics, University of Konstanz, M 677 Konstanz, Germany 
5. Equation (32) should be read as:

$$
\frac{4}{I_{(m)} \alpha_{s} m_{B}^{2}}=\alpha^{\prime} .
$$

6. After equation (37), the boundary condition should be read as: where the function $J(r)$ satisfies the conditions, $J(r) \rightarrow 0$ for $r \rightarrow 0$ or $\infty$ for the stability reasons.

7. Equation (43) should be read as:

$$
J^{(D)}=\left[I_{(m)}+I_{(e)}\right] \frac{\alpha_{s}}{64}\left(m_{B}^{(D)}\right)^{2} R^{2} .
$$

8. Equation (43) should be read as:

$$
4 \frac{\left[I_{(m)}+I_{(e)}\right]^{-1}}{\alpha_{s}\left(m_{B}^{D}\right)^{2}}=\beta .
$$

